\title{
Fuzzy Reliability Based on Hesitant and Dual Hesitant Fuzzy Set Evaluation
}

\author{
Akshay Kumar \\ Department of Mathematics, \\ Graphic Era Hill University, Dehradun, Uttarakhand, India. \\ Corresponding author: akshaykr1001@gmail.com \\ Soni Bisht \\ Department of Mathematics, \\ Alpine Group of Institutes, Dehradun, Uttarakhand, India. \\ E-mail: bsdipi1992@gmail.com \\ Nupur Goyal \\ Department of Mathematics, \\ Graphic Era (Deemed to be University), Dehradun, Uttarakhand, India. \\ E-mail: nupurgoyalgeu@gmail.com \\ Mangey Ram \\ Department of Mathematics; Computer Science and Engineering, \\ Graphic Era (Deemed to be University), Dehradun, Uttarakhand, India. \\ E-mail: drmrswami@geu.ac.in
}

(Received January 15, 2020; Accepted April 27, 2020)

\begin{abstract}
In this study, we calculate hesitant and dual hesitant fuzzy reliability of linear and circular consecutive 2-out-of-4:G system from reliability function and Weibull distribution. After that, we introduced some basic methods for investigating the fuzzy reliability in the form of upper and lower membership and non- membership function having an aggregation operator with equal weights. At last, two numerical examples are also illustrated with the considered technique.
\end{abstract}

Keywords- Fuzzy reliability, Hesitant fuzzy, Dual hesitant fuzzy, Triangular fuzzy number, Linear and circular consecutive $k$-out-of- $n$ :G system.

\section{Introduction}

In recent years, the theory of fuzzy set is widely used in the field of reliability which is based on real-life engineering systems and management. Various researchers used different types of fuzzy set having triangular and trapezoidal fuzzy numbers such as intuitionistic fuzzy, hesitant fuzzy, bifuzzy and picture, etc. for the analysis of fuzzy reliability. Zadeh (1965) explained the relationship between set theory and fuzzy set theory having membership grade zero and one. The author discussed the various operations in the perspective of fuzzy set theory like union, intersection, multiplication, and division, etc. and many more applications in the real world. Atanassov (1983) discussed the intuitionistic fuzzy theory based on the crisp set and define membership and non-membership functions. The author described various properties based on crisp theory and also defined operations as a compliment, union, intersection, multiplication, and division. Torra and Narukawa (2009) described the extension of fuzzy set theory based on 
International Journal of Mathematical, Engineering and Management Sciences

Vol. 6, No. 1, 166-179, 2021

https://doi.org/10.33889/IJMEMS.2021.6.1.010

intuitionistic fuzzy called hesitant fuzzy having membership function value between zero and one. The authors also defined various types of operations and implementation of the crisp set for interval membership. Zhang (2013) first introduced the various power aggregations operators defined on extended hesitant fuzzy set and explained the relationship between properties and operations of hesitant fuzzy which utilized decision-making problems. Rodríguez et al. (2014) introduced hesitant fuzzy set deals with some uncertainty of various kinds of problems and also discussed membership grade. Hao et al. (2017) determined the dual hesitant set of membership and non-membership grade which depend on intuitionistic fuzzy set theory and defined some basic laws of operations on aggregation operators to improve the results.

Liu et al. (2007) calculated the fuzzy reliability and mean time to failure (MTTF) of seriesparallel, parallel-series, and cold standby repairable systems. The authors also described various results, how to calculate the fuzzy reliability of the un-repairable system of lifetime random variables. Kumar et al. (2013) discussed the fundamentals of the fuzzy and intuitionistic fuzzy set having membership and non-membership failure rate of series and parallel systems. The authors extended the idea of the fuzzy set by the idea of intuitionistic fuzzy set and also discussed a new idea to generate the membership and non-membership functions of the fuzzy reliability. Kumar and Ram (2018) also calculated the hesitant fuzzy of the proposed system from aggregation and Weibull distribution in the form of interval fuzzy. Kumar and Ram (2019) evaluated the fuzzy reliability of some basic environmental system as series, parallel and combinations of these systems based on dual hesitant fuzzy set and Weibull distribution. The authors used the concept of the Markov chain technique for evaluating the system reliability in the form of membership and non-membership function. Kumar et al. (2019 and 2020) determined the fuzzy reliability of the considered consecutive-u-out-of- $v$ : F system having non-identical components from the Markov chain process and determined interval fuzzy from intuitionistic and hesitant fuzzy set both membership and non-membership function. Negi and Singh (2019) computed fuzzy reliability and fuzzy MTTF of linear $m$-consecutive weighted- $u$-out-of- $r$-from- $v$ : F system based on universal generating function and exponentially distributed.

From the above discussion, it is clear that many researchers analyzed the reliability of various systems series, parallel and complex systems using fuzzy intervals like fuzzy theory, intuitionistic, bifuzzy, interval-valued fuzzy, hesitant fuzzy and dual hesitant fuzzy, etc. Keeping the above facts in the view, in the present work we have evaluated the hesitant and dual hesitant fuzzy using aggregation operator of linear and circular consecutive-2-out-of-4 using reliability function and Weibull distribution.

\section{Definitions}

\subsection{Fuzzy Set}

The relation between the fuzzy set theory and the crisp set theory was first discussed by Zadeh (1965) based on its membership function of $\alpha$-cut set. A fuzzy set $\mathrm{Z}$ is to describe the membership grade $\mu_{Z}: A \rightarrow[0,1]$, where $\mu_{Z}(A)$ belongs to membership component $z$ in a fuzzy $A$ for all $a \in A$ and $A=\left(a_{1}, a_{2}, \ldots . ., a_{n}\right)$ is the non-empty set of class. 
International Journal of Mathematical, Engineering and Management Sciences

Vol. 6, No. 1, 166-179, 2021

https://doi.org/10.33889/IJMEMS.2021.6.1.010

\subsection{Intuitionistic Fuzzy Set}

The fuzzy set theory defined from the membership and non-membership grade having their $\alpha$ and $\beta$-cut set is called intuitionistic fuzzy set theory is given by Atanassov (1983). It is defined as a set $C$ to be a subset of $b$ as:

$C=\left\{<b, \mu_{C}(b), v_{C}(b)>: c<C\right\}$

where, $\mu_{C}: b \rightarrow[0,1]$ and $v_{C}: b \rightarrow[0,1]$ with $\mu_{C}(b)+v_{C}(b) \leq 1, \forall b \in B$.

\subsection{Hesitant Fuzzy Set}

The hesitant fuzzy set is the extended form of the fuzzy set theory discussed by Torra and Naukara (2009) of a crisp set. The hesitant fuzzy set $B$ based on membership function such as

$$
B=\left\{\left(y, h_{B}(a)\right) / a \in A\right\}
$$

where, $h_{B}(a)$ is the set of the value [0,1] and component $a \in A$ shows the membership grade of the set $B$ and $h$ is a hesitant fuzzy component of the set.

$\mathrm{Xia}$ and $\mathrm{Xu}(2011)$ discussed the functional of the hesitant fuzzy component are as:

(i) $h^{\alpha}=\bigcup_{\beta \in h}\left\{\delta^{\alpha}\right\}, \alpha>0$,

(ii) $\alpha h=\bigcup_{\beta \in h}\left\{1-(1-\beta)^{\alpha}\right\}, \alpha>0$,

(iii) $h_{1} \oplus h_{2}=\bigcup_{\beta_{1} \in h_{1}, \beta_{2} \in h_{2}} \operatorname{Max}\left\{\beta_{1}+\beta_{2}-\beta_{1} \beta_{2}\right\}$,

(iv) $h_{1} \otimes h_{2}=\bigcup_{\beta_{1} \in h_{1}, \beta_{2} \in h_{2}} \operatorname{Min}\left\{\beta_{1} \beta_{2}\right\}$.

Basic functional of hesitant fuzzy components, (Torra, 2010) as

(i) $h^{c}=\bigcup_{\beta \in h}\{1-\beta\}$,

(ii) $h_{1} \cup h_{2}=\bigcup_{\beta_{1} \in h_{1}, \beta_{2} \in h_{2}} \operatorname{Max}\left\{\beta_{1} \beta_{2}\right\}$,

(iii) $h_{1} \cap h_{2}=\bigcup_{\beta_{1} \in h_{1}, \beta_{2} \in h_{2}} \operatorname{Min}\left\{\beta_{1} \beta_{2}\right\}$.

\subsection{Dual Hesitant Fuzzy Set}

The concept of dual hesitant fuzzy set (DHFS) was discussed by Zhu et al. (2012), Chen and Huang (2017) presented the membership and non-membership grade. Let us consider $A$ be a set of DHFS, then $A$ be defined as:

$D_{h}=\{\langle a, C(a), E(a)\rangle a \in A\}$,

where, $C(a)$ and $E(a)$ are sets which values lie in [0,1] and shows the possible membership and non-membership function of the component $a \in A$ to the set $D_{h}$ under valid operations.

$0 \leq \alpha, \beta \leq 1,0 \leq \alpha+\beta \leq 1$, 
International Journal of Mathematical, Engineering and Management Sciences

Vol. 6, No. 1, 166-179, 2021

https://doi.org/10.33889/IJMEMS.2021.6.1.010

where, $\alpha \in C(a), \beta \in E(a), \alpha \in C(a)=\cup_{\alpha \in C(a)} \max \{\alpha\} \quad$ and $\beta \in E(a)=\cup_{\beta \in E(a)}$ for all $a \in A$.

\subsection{Operations on DHFS}

If $D_{h_{1}}$ and $D_{h_{2}}$ two set DHFS then

(i) $D_{h_{1}} \cup D_{h_{2}}=\left\{C \in\left(C_{1} \cup C_{2}\right)\left|C \geq \max \left(C_{1}, C_{2}\right), E \in\left(E_{1}, E_{2}\right)\right| E \leq \min \left(E_{1}, E_{2}\right)\right\}$,

(ii) $D_{h_{1}} \cap D_{h_{2}}=\left\{C \in\left(C_{1} \cap C_{2}\right)\left|C \leq \min \left(C_{1}, C_{2}\right), E \in\left(E_{1}, E_{2}\right)\right| E \geq \max \left(E_{1}, E_{2}\right)\right\}$,

(iii) $D^{c}=\left\{\begin{array}{l}\cup_{\alpha \in C, \beta \in E}\{\{\alpha\},\{\beta\}\}, \text { if } E \neq \gamma, C \neq \gamma \\ \cup_{\alpha \in h}\{\{1-\alpha\},\{\gamma\}\}, \text { if } E=\gamma, C \neq \gamma \\ \cup_{\beta \in E}\{\{\gamma\},\{1-\alpha\}\}, \text { if } C=\gamma, E \neq \gamma .\end{array}\right.$

\section{Triangular Fuzzy Number}

(a) Triangular fuzzy number $\chi$ of lower, upper and modal value is a real value function $R$ basis on the membership grade function $\mu_{\chi}: R \rightarrow[0,1],($ Chang, 1996) as

$$
\mu_{\chi}(z)=\left\{\begin{array}{l}
\frac{x-\chi^{L}}{\chi^{M}-\chi^{L}}, z \in\left[\chi^{L}, \chi^{M}\right] \\
\frac{x-\chi^{U}}{\chi^{M}-\chi^{U}}, z \in\left[\chi^{M}, \chi^{U}\right] \\
0 \quad \text { otherwise }
\end{array}\right.
$$

where, $\chi_{L} \leq \chi_{M} \leq \chi_{U}$.

If we have two triangular fuzzy numbers $\chi_{1}=\left(\chi_{L_{1}}, \chi_{M_{1}}, \chi_{U_{1}}\right)$ and $\chi_{2}=\left(\chi_{L_{2}}, \chi_{M_{2}}, \chi_{U_{2}}\right)$, and these are some operators which are expressed as:

(i) $\chi_{1} \oplus \chi_{2}=\left(\chi_{L_{1}}, \chi_{M_{1}}, \chi_{U_{1}}\right) \oplus\left(\chi_{L_{2}}, \chi_{M_{2}}, \chi_{U_{2}}\right)=\left(\chi_{L_{1}}+\chi_{L_{2}}, \chi_{M_{1}}+\chi_{M_{2}}, \chi_{U_{1}}+\chi_{U_{2}}\right)$,

(ii) $\chi_{1} \otimes \chi_{2}=\left(\chi_{L_{1}}, \chi_{M_{1}}, \chi_{U_{1}}\right) \otimes\left(\chi_{L_{2}}, \chi_{M_{2}}, \chi_{U_{2}}\right)=\left(\chi_{L_{1}} \chi_{L_{2}}, \chi_{M_{1}} \chi_{M_{2}}, \chi_{U_{1}} \chi_{U_{2}}\right)$,

(iii) $\chi_{1}=\chi_{2} \Leftrightarrow \chi_{L_{1}}=\chi_{L_{2}}, \chi_{M_{1}}=\chi_{M_{2}}, \chi_{U_{1}}=\chi_{U_{2}}$,

(iv) $\chi_{1} \leq \chi_{2} \Leftrightarrow \chi_{L_{1}} \leq \chi_{L_{2}}, \chi_{M_{1}} \leq \chi_{M_{2}}, \chi_{U_{1}} \leq \chi_{U_{2}}$,

(v) $\beta \cdot \chi_{1}=(\beta, \beta, \beta)\left(\chi_{L_{1}}, \chi_{M_{1}}, \chi_{U_{1}}\right)=\left(\beta \chi_{L_{1}}, \beta \chi_{M_{1}}, \beta \chi_{U_{1}}\right), \beta>0, \beta \in R$,

(vi) $\chi_{1}^{-1}=\left(\chi_{L_{1}}, \chi_{M_{1}}, \chi_{U_{1}}\right)^{-1}=\left(1 / \chi_{U_{1}}, 1 / \chi_{M_{1}}, 1 / \chi_{U_{1}} 1 / \chi_{L_{1}}\right)$. 
(b) Let us consider $g_{i}(i=1,2, \ldots, n)$ be a class of hesitant fuzzy component and $W=\left(w^{1}, w^{2}, \ldots w^{n}\right)^{t}$ be define weight vector of $g_{i}(i=1,2, \ldots, n)$ belongs $W^{i} \in[0,1]$ and $\sum_{i=1}^{n} W^{i}=1$, then weighted averaging operator also called score function of a triangular fuzzy set is

$$
\begin{aligned}
& G^{n} \rightarrow G=\left(g_{1}, g_{2}, \ldots, g_{n}\right)={\underset{\Theta}{i}}_{i}^{n}\left(W^{i} g_{i}\right) \\
& =\left\{\left(1-\prod_{i=1}^{n}\left(1-\chi_{L_{i}}\right)^{W^{i}}, 1-\prod_{i=1}^{n}\left(1-\chi_{M_{i}}\right)^{W^{i}}, 1-\prod_{i=1}^{n}\left(1-\chi_{U_{i}}\right)^{W^{i}}\right)\right\}
\end{aligned}
$$

\section{Fuzzy Weibull Distribution}

In the case of reliability evaluation, Weibull distribution is generally used with hazard function and lifetime components are as follows:

$f(t)=\left\{\begin{array}{ll}\beta \theta^{-1}\left(t \theta^{-1}\right)^{\beta-1} e^{\left\{-\left(t \theta^{-1}\right)^{\beta}\right.}, & x>0, \theta>0, \beta>0 \\ 0, & \text { otherwise }\end{array}\right\}, \quad$ namely, $\theta=$ scale and $\beta=$ shape parameter.

Hence, the reliability function of the system having Weibull distribution is

$$
=\mathrm{e}^{\left\{\left(\frac{x-\eta}{\theta}\right)^{\beta}\right\}}
$$

Here, $\eta=0$ the failure rate $=e^{\left\{-\left(x \theta^{-1}\right)^{\beta}\right\}}$.

\section{Linear and Circular Consecutive $u$-out-of- $v$ System}

Linear and circular consecutive system are two types such as linear and circular consecutive $u$ out-of- $v: F$ and G systems, if at least consecutive $u$ component failed then it is called failed system and if at least consecutive $u$ components work it called working system respectively. The system components have two conditions either working or failed. These are some applications of the proposed system as Vacuum systems in accelerators and field of integrated circuits. If all components of the system having equal reliability i.e. $R_{l}=R_{2}=R_{3}=\ldots R_{v}=R$ then, reliability and unreliability function of the linear and circular consecutive $u$-out-of- $v$ :G system can be determined as:

$$
\begin{aligned}
& p(v ; u)=p(v-1 ; u)+k r^{k}(1-p(v-u-1 ; u)) \text { and } \\
& q(v, u)=\sum_{t=v-u+1}^{v} \sum_{m=1}^{(t+u) v} k^{2} r^{v-t+m-1} \cdot q(t-m-1, u)
\end{aligned}
$$


International Journal of Mathematical, Engineering and Management Sciences

Vol. 6, No. 1, 166-179, 2021

https://doi.org/10.33889/IJMEMS.2021.6.1.010

also calculate the reliability of circular consecutive- $u$-out-of- $v: \mathrm{G}$ system $q(i ; j)=1-q((i, j) ; u)$ (Zhang, 1988).

\subsection{Reliability Function of Linear Consecutive 2-out-of-4:G System}

Consider a linear consecutive 2-out-of-4: $\mathrm{G}$ system having the same reliability components and evaluate the reliability function of the system as:

First, we assess the reliability function using the formula of linear consecutive 2-out-of-4:G system see (Zhang, 1988) as:

$$
p(n ; k)=p(n-1 ; k)+u r^{k}(1-r(n-k-1 ; k))
$$

Taking $n=4$ and $k=2$, put in Eq. (4), then we can express as:

$$
\begin{aligned}
& p(0,2)=p(-1,2)+q(-3,2)=0 \\
& p(1,2)=0 \\
& p(2,2)=r^{2} \\
& p(3,2)=2 r^{2}-r^{3} \\
& p(4,2)=3 r^{2}-2 r^{3}
\end{aligned}
$$

\subsection{Reliability Function of Circular Consecutive 2-out-of-4:G System}

Using Eq. (4), to estimate the reliability of circular consecutive 2-out-of-4:G system same as a previous linear system, now we will start from Eq. (5) to get unreliability and reliability function such as:

$$
p(4,2)=3 r^{2}-2 r^{3}
$$

Using the formula (Zhang, 1988) for $v=4$ and $u=2$ we have,

$$
q(v, u)=\sum_{t=v-u+1}^{v} \sum_{m=1}^{(t+u) v} u^{2} r^{v-t+m-1} \otimes q(t-m-1, u),
$$

then,

$$
\begin{aligned}
& q(4,2)=\sum_{t=3}^{4} \sum_{m=1}^{(t+2)} u^{2} r^{3-t+m} \otimes q(t-m-1,2) \\
& =\sum_{m=1}^{1} u^{2} r^{m} q(2-m, 2)+\sum_{m=1}^{2} u^{2} r^{m-1} q(3-m, 2) \\
& \left.=u^{2}[2 r q(1,2)+q(2,2))\right] \\
& =u^{2}[2 r(1-p(1,2)+1-p(2,2)] \\
& q(4,2)=1+4 r^{3}-4 r^{2}-r^{4}, \text { hence }
\end{aligned}
$$


International Journal of Mathematical, Engineering and Management Sciences

Vol. 6, No. 1, 166-179, 2021

https://doi.org/10.33889/IJMEMS.2021.6.1.010

$$
\begin{aligned}
& p(4,2)=1-q(4,2) \\
& p(4,2)=4 r^{2}-4 r^{3}+r^{4}
\end{aligned}
$$

\section{Numerical Example}

We considered the hesitant membership function $\phi$ from a triangular fuzzy number of lifetime distribution are obtained, if membership function defined as $\varphi=\varphi_{1} ;(1.5,2,2.5)$, $\varphi_{2} ;(1.75,1.85,1.95), \varphi_{3} ;(2.25,2.5,2.75)$ having hesitant fuzzy number within the same weightage given $W_{l}=W_{2}=W_{3}=1 / 3$, hence $\alpha$-cut is described as: $\phi=\phi_{1} ;(1.5+0.5 \alpha, 2.5-0.5 \alpha)$, $\phi_{2} ;(1.75+0.10 \alpha, 1.95-0.10 \alpha), \phi_{3} ;(2.25+0.25 \alpha, 2.75-0.25 \alpha)$.

Now with the help of Eqs. (3), (5) and (6), we obtain fuzzy reliability of linear and circular consecutive 2-out-of-4: $\mathrm{G}$ system for all $\alpha$, taking $\beta=0.5$ and $t=5$ is

$$
\begin{aligned}
& R(t)(\alpha)=3 \mathrm{e}^{\left(-2\left(\frac{t}{1.5+0.5 \alpha}\right)^{\beta}\right)}-2 \mathrm{e}^{\left(-3\left(\frac{t}{1.5+0.5 \alpha}\right)^{\beta}\right)}, \\
& 3 \mathrm{e}^{\left(-2\left(\frac{t}{2.5-0.5 \alpha}\right)^{\beta}\right)}-2 \mathrm{e}^{\left(-3\left(\frac{t}{2.5-0.5 \alpha}\right)^{\beta}\right)} ; \\
& 3 \mathrm{e}^{\left(-2\left(\frac{t}{1.75+0.10 \alpha}\right)^{\beta}\right)}-2 \mathrm{e}^{\left(-3\left(\frac{t}{1.75+0.10 \alpha}\right)^{\beta}\right)}, \\
& 3 \mathrm{e}^{\left(-2\left(\frac{t}{1.95-0.10 \alpha}\right)^{\beta}\right)}-2 \mathrm{e}^{\left(-3\left(\frac{t}{1.95-0.10 \alpha}\right)^{\beta}\right)} \\
& 3 \mathrm{e}^{\left(-2\left(\frac{t}{2.25+0.25 \alpha}\right)^{\beta}\right)}-2 \mathrm{e}^{\left(-3\left(\frac{t}{2.25+0.25 \alpha}\right)^{\beta}\right)}, \\
& 3 \mathrm{e}^{\left(-2\left(\frac{t}{2.75-0.25 \alpha}\right)^{\beta}\right)}-2 \mathrm{e}^{\left(-3\left(\frac{t}{2.75-0.25 \alpha}\right)^{\beta}\right)} ; \\
& R(t)(\alpha)=4 \mathrm{e}^{\left(-2\left(\frac{t}{1.5+0.5 \alpha}\right)^{\beta}\right)}-4 \mathrm{e}^{\left(-3\left(\frac{t}{1.5+0.5 \alpha}\right)^{\beta}\right)}+\mathrm{e}^{\left(-4\left(\frac{t}{1.5+0.5 \alpha}\right)^{\beta}\right)}, \\
& 4 \mathrm{e}^{\left(-2\left(\frac{t}{2.5-0.5 \alpha}\right)^{\beta}\right)}-4 \mathrm{e}^{\left(-3\left(\frac{t}{2.5-0.5 \alpha}\right)^{\beta}\right)}+\mathrm{e}^{\left(-4\left(\frac{t}{2.5-0.5 \alpha}\right)^{\beta}\right)} ; \\
& 4 \mathrm{e}^{\left(-2\left(\frac{t}{1.75+0.10 \alpha}\right)^{\beta}\right)}-4 \mathrm{e}^{\left(-3\left(\frac{t}{1.75+0.10 \alpha}\right)^{\beta}\right)}+\mathrm{e}^{\left(-4\left(\frac{t}{1.75+0.10 \alpha}\right)^{\beta}\right)}, \\
& 4 \mathrm{e}^{\left(-2\left(\frac{t}{1.95-0.10 \alpha}\right)^{\beta}\right)}-4 \mathrm{e}^{\left(-3\left(\frac{t}{1.95-0.10 \alpha}\right)^{\beta}\right)}+\mathrm{e}^{\left(-4\left(\frac{t}{1.95-0.10 \alpha}\right)^{\beta}\right)} ; \\
& 4 \mathrm{e}^{\left(-2\left(\frac{t}{2.25+0.25 \alpha}\right)^{\beta}\right)}-4 \mathrm{e}^{\left(-3\left(\frac{t}{2.25+0.25 \alpha}\right)^{\beta}\right)}+\mathrm{e}^{\left(-4\left(\frac{t}{2.25+0.25 \alpha}\right)^{\beta}\right)}, \\
& 4 \mathrm{e}^{\left(-2\left(\frac{t}{2.75-0.25 \alpha}\right)^{\beta}\right)}-4 \mathrm{e}^{\left(-3\left(\frac{t}{2.75-0.25 \alpha}\right)^{\beta}\right)}+\mathrm{e}^{\left(-4\left(\frac{t}{2.75-0.25 \alpha}\right)^{\beta}\right)} .
\end{aligned}
$$


International Journal of Mathematical, Engineering and Management Sciences

Vol. 6, No. 1, 166-179, 2021

https://doi.org/10.33889/IJMEMS.2021.6.1.010

Hence, Table 1, Table 2 and Figures 1-6 discussed the fuzzy reliability of considered system based on reliability function and hesitant fuzzy membership grade using the above equations are as:

Table 1. Reliability of the linear system.

\begin{tabular}{|c|c|c|c|}
\hline$\alpha$-cut & $\boldsymbol{R}_{1}[\alpha]$ & $\boldsymbol{R}_{2}[\alpha]$ & $\boldsymbol{R}_{3}[\alpha]$ \\
\hline $\mathbf{0}$ & $(0.0778,0.1772)$ & $(0.1021,0.1219)$ & $(0.1521,0.2020)$ \\
\hline $\mathbf{0 . 1}$ & $(0.0826,0.1772)$ & $(0.1030,0.1209)$ & $(0.1546,0.1996)$ \\
\hline $\mathbf{0 . 2}$ & $(0.0874,0.1671)$ & $(0.1040,0.1199)$ & $(0.1596,0.1946)$ \\
\hline $\mathbf{0 . 3}$ & $(0.0923,0.1621)$ & $(0.1050,0.1189)$ & $(0.1621,0.1921)$ \\
\hline $\mathbf{0 . 4}$ & $(0.0971,0.1571)$ & $(0.1060,0.1179)$ & $(0.1646,0.1896)$ \\
\hline $\mathbf{0 . 5}$ & $(0.1021,0.1521)$ & $(0.1070,0.1169)$ & $(0.1671,0.1871)$ \\
\hline $\mathbf{0 . 6}$ & $(0.1070,0.1470)$ & $(0.1080,0.1159)$ & $(0.1722,0.1822)$ \\
\hline $\mathbf{0 . 7}$ & $(0.1120,0.1420)$ & $(0.1090,0.1149)$ & $(0.1747,0.1797)$ \\
\hline $\mathbf{0 . 8}$ & $(0.1169,0.1370)$ & $(0.1100,0.1139)$ & $(0.1772,0.1772)$ \\
\hline $\mathbf{0 . 9}$ & $(0.1219,0.1320)$ & $(0.1110,0.1129)$ & $(0.1120,0.1120)$ \\
\hline $\mathbf{1}$ & $(0.1269,0.1269)$ & & \\
\hline
\end{tabular}

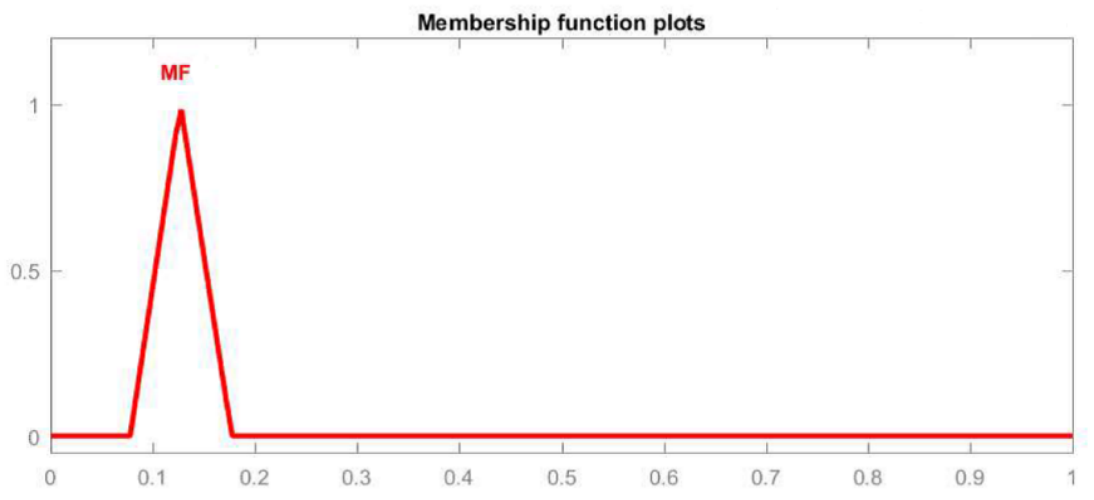

Figure 1. Membership function of $R_{1}(\alpha)$ for linear system.

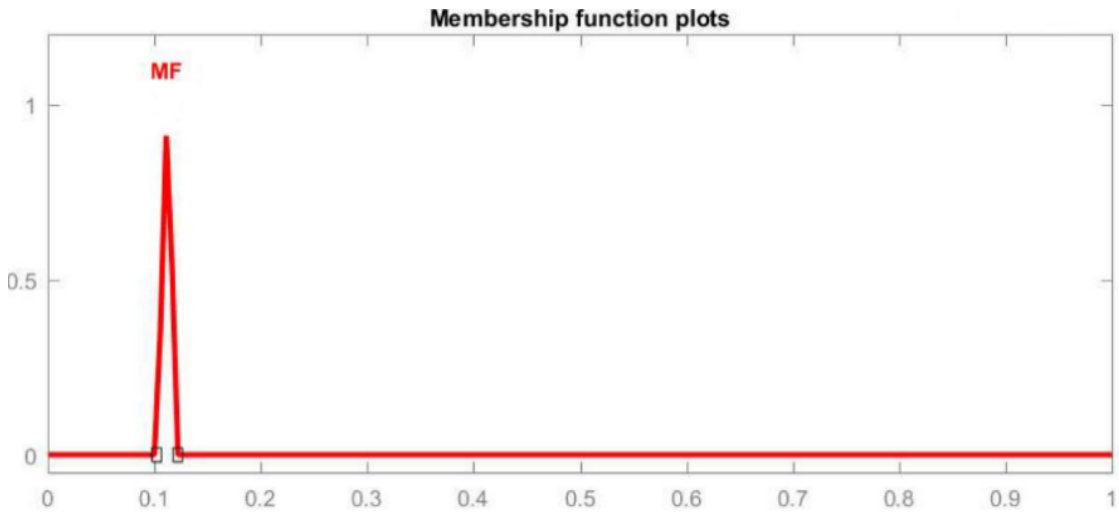

Figure 2. Membership function of $R_{2}(\alpha)$ for linear system. 
International Journal of Mathematical, Engineering and Management Sciences

Vol. 6, No. 1, 166-179, 2021

https://doi.org/10.33889/IJMEMS.2021.6.1.010

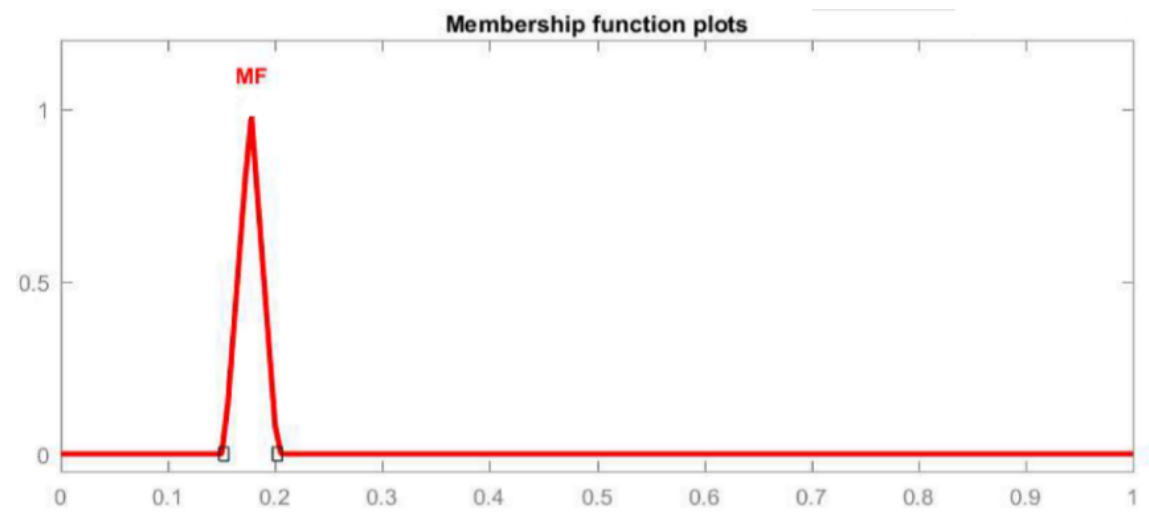

Figure 3. Membership function of $R_{3}(\alpha)$ for linear system.

Table 2. Reliability of the consecutive system.

\begin{tabular}{|c|c|c|c|}
\hline$\alpha$-cut & $\boldsymbol{R}_{1}[\alpha]$ & $\boldsymbol{R}_{2}[\alpha]$ & $\boldsymbol{R}_{3}[\alpha]$ \\
\hline $\mathbf{0}$ & $(0.0878,0.1824)$ & $(0.1122,0.1315)$ & $(0.1598,0.2042)$ \\
\hline $\mathbf{0 . 1}$ & $(0.0926,0.1780)$ & $(0.1131,0.1305)$ & $(0.1621,0.2021)$ \\
\hline $\mathbf{0 . 2}$ & $(0.0975,0.1735)$ & $(0.1141,0.1296)$ & $(0.1644,0.1999)$ \\
\hline $\mathbf{0 . 3}$ & $(0.1024,0.1689)$ & $(0.1151,0.1286)$ & $(0.1689,0.1978)$ \\
\hline $\mathbf{0 . 4}$ & $(0.1073,0.1644)$ & $(0.1160,0.1276)$ & $(0.1712,0.1934)$ \\
\hline $\mathbf{0 . 5}$ & $(0.1122,0.1598)$ & $(0.1170,0.1267)$ & $(0.1735,0.1913)$ \\
\hline $\mathbf{0 . 6}$ & $(0.1170,0.1551)$ & $(0.1180,0.1257)$ & $(0.1757,0.1891)$ \\
\hline $\mathbf{0 . 7}$ & $(0.1219,0.1505)$ & $(0.1190,0.1248)$ & $(0.1780,0.1869)$ \\
\hline $\mathbf{0 . 8}$ & $(0.1267,0.1458)$ & $(0.1199,0.1238)$ & $(0.1802,0.1847)$ \\
\hline $\mathbf{0 . 9}$ & $(0.1315,0.1410)$ & $(0.1209,0.1228)$ & \\
\hline $\mathbf{1}$ & $(0.1363,0.1363)$ & $(0.1219,0.1219)$ & \\
\hline
\end{tabular}

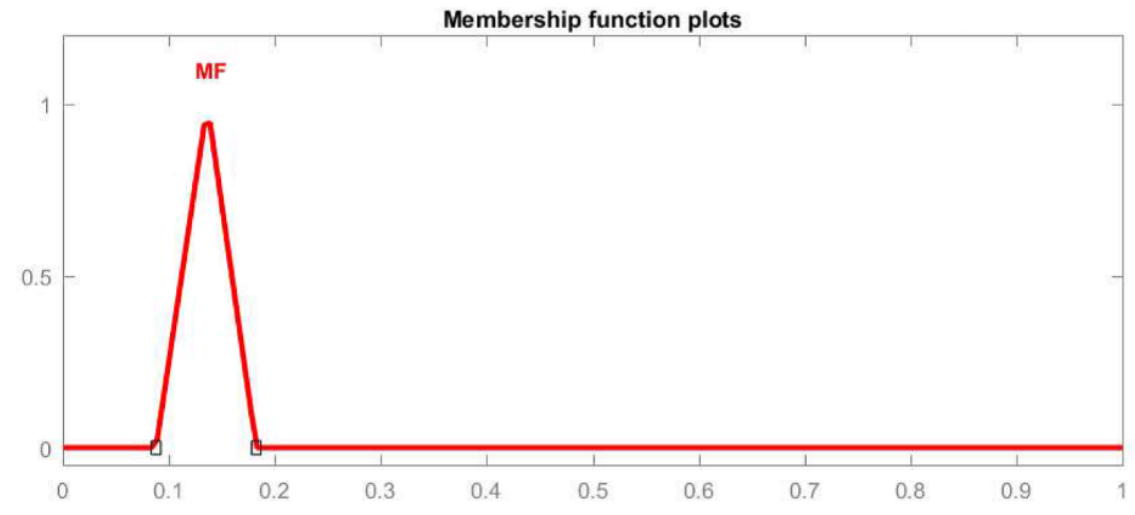

Figure 4. Membership function of $R_{1}(\alpha)$ for consecutive system. 
International Journal of Mathematical, Engineering and Management Sciences

Vol. 6, No. 1, 166-179, 2021

https://doi.org/10.33889/IJMEMS.2021.6.1.010

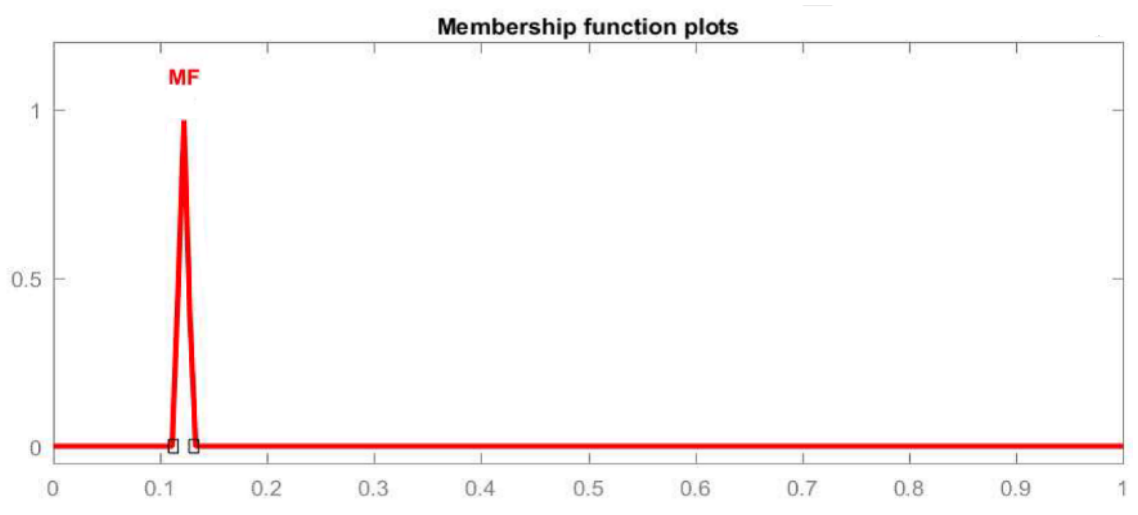

Figure 5. Membership function of $R_{2}(\alpha)$ for consecutive system.

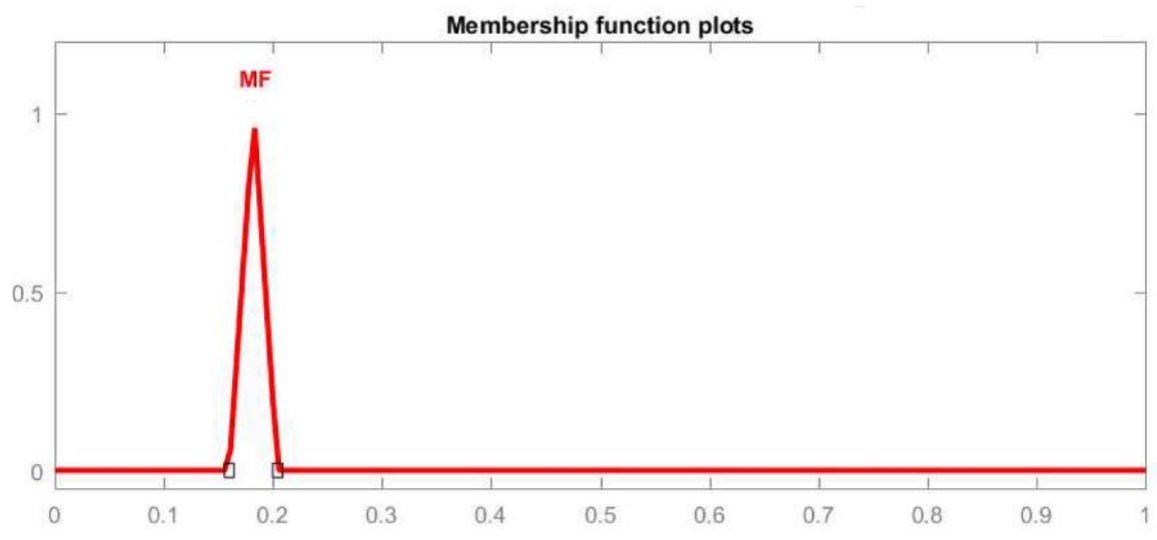

Figure 6. Membership function of $R_{3}(\alpha)$ for consecutive system.

Now, we have calculated hesitant fuzzy by using of aggregation operator from Eq. (2), and obtained the results for the considered system as shown in Table 3 and Table 4, correspondingly Figure 7 and Figure 8, show the triangular membership function.

Table 3. Reliability of the linear system after aggregation operator.

\begin{tabular}{|c|c|}
\hline$\alpha$-cut & $\boldsymbol{R}_{\mathbf{1}}[\boldsymbol{\alpha}]$ \\
\hline $\mathbf{0}$ & $(0.1112,0.1677)$ \\
\hline $\mathbf{0 . 1}$ & $(0.1139,0.1665)$ \\
\hline $\mathbf{0 . 2}$ & $(0.1146,0.1620)$ \\
\hline $\mathbf{0 . 3}$ & $(0.1195,0.1597)$ \\
\hline $\mathbf{0 . 4}$ & $(0.1222,0.1562)$ \\
\hline $\mathbf{0 . 5}$ & $(0.1250,0.1534)$ \\
\hline $\mathbf{0 . 6}$ & $(0.1278,0.1488)$ \\
\hline $\mathbf{0 . 7}$ & $(0.1307,0.1477)$ \\
\hline $\mathbf{0 . 9}$ & $(0.1335,0.1448)$ \\
\hline $\mathbf{1}$ & $(0.1363,0.1420)$ \\
\hline
\end{tabular}


International Journal of Mathematical, Engineering and Management Sciences

Vol. 6, No. 1, 166-179, 2021

https://doi.org/10.33889/IJMEMS.2021.6.1.010

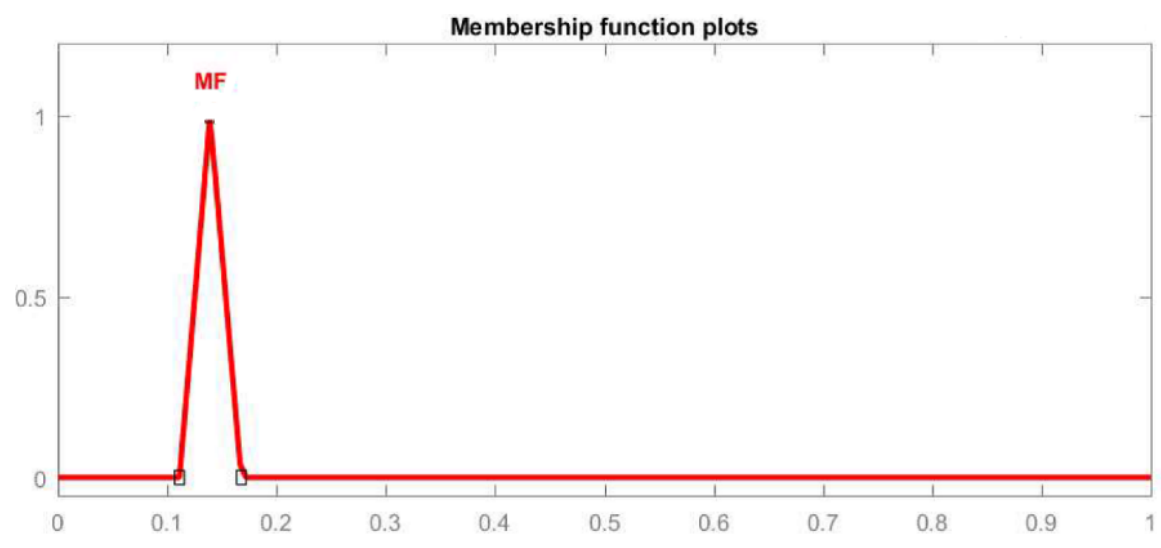

Figure 7. Membership function of linear consecutive 2-out-4: G system.

Table 4. Reliability of the circular consecutive system after aggregation operator.

\begin{tabular}{|c|c|}
\hline $\boldsymbol{\alpha}$-cut & $\boldsymbol{R}_{\mathbf{1}}[\boldsymbol{\alpha}]$ \\
\hline $\mathbf{0}$ & $(0.1204,0.1733)$ \\
\hline $\mathbf{0 . 1}$ & $(0.1231,0.1707)$ \\
\hline $\mathbf{0 . 2}$ & $(0.1258,0.1673)$ \\
\hline $\mathbf{0 . 3}$ & $(0.1285,0.1656)$ \\
\hline $\mathbf{0 . 4}$ & $(0.1312,0.1630)$ \\
\hline $\mathbf{0 . 5}$ & $(0.1339,0.1604)$ \\
\hline $\mathbf{0 . 6}$ & $(0.1366,0.1578)$ \\
\hline $\mathbf{0 . 7}$ & $(0.1393,0.1552)$ \\
\hline $\mathbf{0 . 9}$ & $(0.1419,0.1526)$ \\
\hline $\mathbf{1}$ & $(0.1446,0.1499)$ \\
\hline & \\
\hline
\end{tabular}

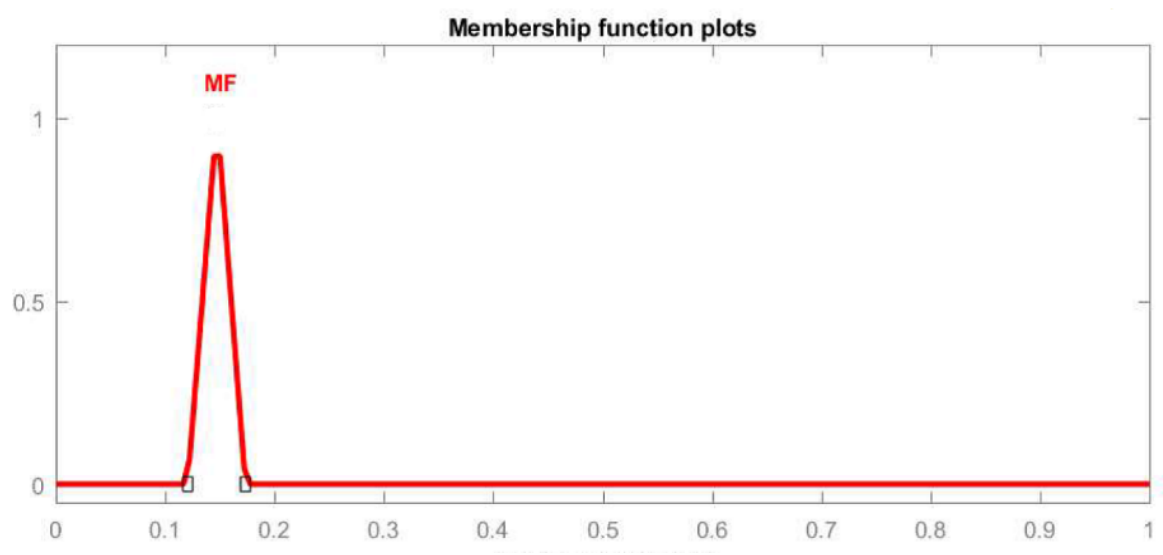

Figure 8. Membership function of circular consecutive 2-out-4: G system. 
International Journal of Mathematical, Engineering and Management Sciences

Vol. 6, No. 1, 166-179, 2021

https://doi.org/10.33889/IJMEMS.2021.6.1.010

\subsection{Numerical Illustration}

The fuzzy reliability of linear and circular consecutive 2-out-4: G system is obtained from the dual hesitant fuzzy set and triangular fuzzy number with Weibull distribution. If triangular fuzzy is defined as $s=(1,1.25,1.50,1.75,2)$ and $\alpha$-cut of dual hesitant fuzzy number is (Chen and Huang, 2017) as

$R(t)[\alpha]=\max (1+0.5 \alpha, 1.25+0.5(1-\alpha)) ; \min (2+0.5 \alpha, 1.75+0.25(1-\alpha))$.

For all $\alpha$, taking $\beta=0.5$ and $t=5$

$$
\begin{aligned}
& R(t)(\alpha)=\max \left[3 \mathrm{e}^{\left(-2\left(\frac{t}{1+0.5 \alpha}\right)^{\beta}\right)}-2 \mathrm{e}^{\left(-3\left(\frac{t}{1+0.5 \alpha}\right)^{\beta}\right)},\right. \\
& 3 \mathrm{e}^{\left(-2\left(\frac{t}{1.25+0.5(1-\alpha)}\right)^{\beta}\right)}-2 \mathrm{e}^{\left(-3\left(\frac{t}{1.25+0.5(1-\alpha)}\right)^{\beta}\right)} \\
& \min \left[3 \mathrm{e}^{\left(-2\left(\frac{t}{2+0.5 \alpha}\right)^{\beta}\right)}-2 \mathrm{e}^{\left(-3\left(\frac{t}{2+0.5 \alpha}\right)^{\beta}\right)},\right. \\
& \left.3 \mathrm{e}^{\left(-2\left(\frac{t}{1.75+0.25(1-\alpha)}\right)^{\beta}\right)}-2 \mathrm{e}^{\left(-3\left(\frac{t}{1.75+0.25(1-\alpha)}\right)^{\beta}\right)}\right] . \\
& R(t)(\alpha)=\max \left[4 \mathrm{e}^{\left(-2\left(\frac{t}{1+0.5 \alpha}\right)^{\beta}\right)}-4 \mathrm{e}^{\left(-3\left(\frac{t}{1+0.5 \alpha}\right)^{\beta}\right)}+\mathrm{e}^{\left(-4\left(\frac{t}{1+0.5 \alpha}\right)^{\beta}\right)},\right. \\
& \left.4 \mathrm{e}^{\left(-2\left(\frac{t}{1.25+0.5(1-\alpha)}\right)^{\beta}\right)}-4 \mathrm{e}^{\left(-3\left(\frac{t}{1.25+0.5(1-\alpha)}\right)^{\beta}\right)}+\mathrm{e}^{\left(-4\left(\frac{t}{1.25+0.5(1-\alpha)}\right)^{\beta}\right)}\right] \\
& \min \left[4 \mathrm{e}^{\left(-2\left(\frac{t}{2+0.5 \alpha}\right)^{\beta}\right)}-4 \mathrm{e}^{\left(-3\left(\frac{t}{2+0.5 \alpha}\right)^{\beta}\right)}+\mathrm{e}^{\left(-4\left(\frac{t}{2+0.5 \alpha}\right)^{\beta}\right)},\right. \\
& 4 \mathrm{e}^{\left(-2\left(\frac{t}{1.75+0.25(1-\alpha)}\right)^{\beta}\right)}-4 \mathrm{e}^{\left(-3\left(\frac{t}{1.75+0.25(1-\alpha)}\right)^{\beta}\right)}+\mathrm{e}^{\left.\left(-4\left(\frac{t}{1.75+0.25(1-\alpha)}\right)^{\beta}\right)\right]} .
\end{aligned}
$$


International Journal of Mathematical, Engineering and Management Sciences

Vol. 6, No. 1, 166-179, 2021

https://doi.org/10.33889/IJMEMS.2021.6.1.010

Hence, Table 5 and Table 6 show the results of fuzzy reliability based on dual hesitant fuzzy set from Eqs. (1)-(6) of considered system are as:

Table 5. Reliability of the linear system.

\begin{tabular}{|c|c|c|}
\hline $\boldsymbol{\alpha}$-cut & Max $(\boldsymbol{\alpha}, \boldsymbol{\beta})$ & Min $(\boldsymbol{\alpha}, \boldsymbol{\beta})$ \\
\hline $\mathbf{0}$ & $(.0052, .0236)$ & $(.0318, .0318)$ \\
\hline $\mathbf{0 . 1}$ & $(.0061, .0221)$ & $(.0336, .0310)$ \\
\hline $\mathbf{0 . 2}$ & $(.0070, .0206)$ & $.0353, .0301)$ \\
\hline $\mathbf{0 . 3}$ & $(.0079, .0191)$ & $(.0371, .0293)$ \\
\hline $\mathbf{0 . 4}$ & $(.0090, .0177)$ & $(.0389, .0285)$ \\
\hline $\mathbf{0 . 5}$ & $(.0101, .0163)$ & $(.0407, .0276)$ \\
\hline $\mathbf{0 . 6}$ & $(.0112, .0149)$ & $(.0425, .0268)$ \\
\hline $\mathbf{0 . 7}$ & $(.0124, .0137)$ & $.0444, .0260)$ \\
\hline $\mathbf{0 . 8}$ & $(.0137, .0124)$ & $(.0462, .0252)$ \\
\hline $\mathbf{0 . 9}$ & $(.0149, .0112)$ & $(.0481, .0244)$ \\
\hline $\mathbf{1}$ & $(.0163, .0101)$ & $(.0500, .0236)$ \\
\hline
\end{tabular}

Table 6. Reliability of the circular system.

\begin{tabular}{|c|c|c|}
\hline $\boldsymbol{\alpha}$-cut & Max $(\boldsymbol{\alpha}, \boldsymbol{\beta})$ & Min $(\boldsymbol{\alpha}, \boldsymbol{\beta})$ \\
\hline $\mathbf{0}$ & $(.0069, .0306)$ & $(.0409, .0409)$ \\
\hline $\mathbf{0 . 1}$ & $(.0080, .0286)$ & $(.0431, .0399)$ \\
\hline $\mathbf{0 . 2}$ & $(.0092, .0262)$ & $(.0453, .0388)$ \\
\hline $\mathbf{0 . 3}$ & $(.0104, .0248)$ & $(.0475, .0377)$ \\
\hline $\mathbf{0 . 4}$ & $(.0118, .0230)$ & $(.0498, .0367)$ \\
\hline $\mathbf{0 . 5}$ & $(.0132, .0212)$ & $(.0521, .0356)$ \\
\hline $\mathbf{0 . 6}$ & $(.0146, .0195)$ & $(.0543, .0346)$ \\
\hline $\mathbf{0 . 7}$ & $(.0162, .0178)$ & $(.0567, .0336)$ \\
\hline $\mathbf{0 . 8}$ & $(.0178, .0162)$ & $(.0590, .0326)$ \\
\hline $\mathbf{0 . 9}$ & $(.0195, .0146)$ & $(.0613, .0315)$ \\
\hline $\mathbf{1}$ & $(.0212, .0132)$ & $.0637, .0306)$ \\
\hline
\end{tabular}

\section{Conclusion}

Fuzzy reliability is an essential part of engineering and real-life systems based on uncertainty, there is a different kind of uncertainty of fuzziness for problem formulation. The hesitant and dual hesitant fuzzy approaches to determine the fuzzy reliability of linear and circular consecutive 2out-of-4: G system using aggregation operators, Weibull distribution and reliability function have been discussed. Table 1 and Table 2 show the hesitant fuzzy membership function in figure 1 to figure 6. Table 3 and Table 4 illustrate the hesitant fuzzy after using aggregation operator with the same weight is given $W_{1}=W_{2}=W_{3}=1 / 3$ in figure 7 and figure 8 . In the case of dual hesitant fuzzy, Table 5 and Table 6 demonstrate the membership and non-membership function in the form of maximum and minimum $\alpha$ and $\beta$ cut sets.

\section{Conflict of Interest}

The authors confirm that there is no conflict of interest to declare for this publication.

\section{Acknowledgments}

The authors sincerely thank and appreciate the editor and reviewers for their time and valuable comments. 
International Journal of Mathematical, Engineering and Management Sciences

Vol. 6, No. 1, 166-179, 2021

https://doi.org/10.33889/IJMEMS.2021.6.1.010

\section{References}

Atanassov, K.T. (1983). Intuitionistic fuzzy sets VII ITKR’s Session. Sofia, June, 1, 983.

Chang, D.Y. (1996). Applications of the extent analysis method on fuzzy AHP. European Journal of Operational Research, 95(3), 649-655.

Chen, J., \& Huang, X. (2017). Dual hesitant fuzzy probability. Symmetry, 9(4), 52.

Hao, Z., Xu, Z., Zhao, H., \& Su, Z. (2017). Probabilistic dual hesitant fuzzy set and its application in risk evaluation. Knowledge-Based Systems, 127, 16-28.

Kumar, A., Singh, S.B., \& Ram, M. (2020). Systems reliability assessment using hesitant fuzzy set. International Journal of Operational Research. 38(1), 1-18.

Kumar, A., \& Ram, M. (2019). Reliability analysis for environment systems using dual hesitant fuzzy set. In: Ram, M. (ed) Advanced Fuzzy Logic Approaches in Engineering Science, 162-173. IGI Global.

Kumar, A., \& Ram, M., (2018). System reliability analysis based on weibull distribution and hesitant fuzzy set. International Journal of Mathematical, Engineering and Management Sciences. 3(4), 513-521.

Kumar, A., Singh, S.B., \& Ram, M. (2019). Reliability appraisal for consecutive- $k$-out-of- $n$ : F system of non-identical components with intuitionistic fuzzy set. International Journal of Operational Research, 36(3), 362-374.

Kumar, M., Yadav, S.P., \& Kumar, S. (2013). Fuzzy system reliability evaluation using time-dependent intuitionistic fuzzy set. International Journal of Systems Science, 44(1), 50-66.

Liu, Y., Tang, W., \& Zhao, R. (2007). Reliability and mean time to failure of unrepairable systems with fuzzy random lifetimes. IEEE Transactions on Fuzzy Systems, 15(5), 1009-1026.

Negi, S., \& Singh, S.B. (2019). Fuzzy reliability evaluation of linear $m$-consecutive weighted- $k$-out-of- $r$ from- $n$ : F systems. International Journal of Computing Science and Mathematics, 10(6), 606-621.

Rodríguez, R.M., Martínez, L., Torra, V., Xu, Z.S., \& Herrera, F. (2014). Hesitant fuzzy sets: state of the art and future directions. International Journal of Intelligent Systems, 29(6), 495-524.

Torra, V. (2010). Hesitant fuzzy sets. International Journal of Intelligent Systems, 25(6), 529-539.

Torra, V., \& Narukawa, Y. (2009). On hesitant fuzzy sets and decision. In IEEE International Conference on Fuzzy Systems FUZZY (pp.1378-1382). IEEE, Jeju Island, South Korea.

Xia, M., \& Xu, Z. (2011). Hesitant fuzzy information aggregation in decision making. International Journal of Approximate Reasoning, 52(3), 395-407.

Zadeh, L.A. (1965). Fuzzy sets. Information and Control, 8(3), 338-353.

Zhang, W. (1988). Theory and analysis of consecutive- $k$-out-of- $n: \mathrm{G}$ systems reliability. Retrospective Theses and Dissertations 9749. https://lib.dr.iastate.edu/rtd/9749.

Zhang, Z. (2013). Hesitant fuzzy power aggregation operators and their application to multiple attribute group decision making. Information Sciences, 234, 150-181.

Zhu, B., Xu, Z., \& Xia, M. (2012). Dual hesitant fuzzy sets. Journal of Applied Mathematics, Article ID 879629, 1-13, doi:10.1155/2012/879629. 